\title{
POLITIQUE PISCICOLE POUR LES EAUX CONTINENTALES DE L'OUEST AFRICAIN
}

\author{
par Jacques BARD \\ Inspecteur principal des Eaux et Forêts \\ (Cadre autonome)
}

\section{Production piscicole et économie générale.}

L'intérêt qui s'est manifesté ces dernières années dans l'Ouest Africain pour l'amélioration de la production des eaux continentales est né, semble-t-il, de l'apparition de la pisciculture du Tilapia, si bien qu'assez paradoxalement, c'est la production piscicole artificielle qu a éveillé l'attention sur la production naturelle.

Pêche ou Pisciculture, il s'agit d'une production entièrement artisanale, laquelle peut donner naissance à des courants commerciaux fort importants tels que ceux des poissons séchés et fumés du Niger vers la Côte d'Ivoire, le Ghana et le Togo ou du bassin du Tchad vers la Nigéria et le Nord-Cameroun. Commercialisée ou pas, cette production échappe pour sa plus grande partie à tout contrôle et donc n'alimente guère les recettes budgétaires des pays intéressés. Pratiquement, tout se passe comme s'il s'agissait d'un marché strictement intérieur. Cette considération détourne de la production piscicole l'attention des Pouvoirs publics locaux, absorbés qu'ils sont par les soucis budgétaires immédiats. Pourtant, l'orientation exclusive de l'amélioration de la production vers les produits d'exportation tels que café, cacao, banane, coton pour lesquels les marchés mondiaux touchent assez facilement la limite de saturation, ne saurait être considérée comme suffisante pour assurer l'harmonieuse expansion économique d'un pays; le développement de la production d'aliments de base (dont la production piscicole) est au moins aussi important. Mais son financement apparaît, dans la conjoncture présente, délicat à dégager. Aussi ce financement a-t-il été, jusqu'à présent, fondé sur l'aide extérieure.

\section{Importance relative de la pêche et de la pisciculture.}

Dans leur état naturel, les eaux continentales de l'Ouest africain sont inégalement productives. Il semble, en effet, que la production du poisson soit étroitement liée à l'ensoleillement et aussi à l'alternance des crues et décrues sur une surface suffisante, laquelle crée un véritable 
assolement dans le cycle biologique de l'eau douce. Il en résulte que, si les eaux des zones soudanaises et sahéliennes sont en général très productives, les eaux de la zone de forêt le sont infiniment moins.

D'autre part, dans cette dernière zone spécialement, les cours d'eau sont souvent d'accès difficile et leurs bords insalubres : les pêcheurs sont donc rares. La population est sollicitée par des activités agricoles dont le siège se situe le plus souvent loin des rivières.

Quant aux petits cours d'eau, trop ombragés, donc peu productifs, l'usage de la pêche au poison les a, de surplus, largement dépeuplés.

Le développement de la production piscicole d'un pays doit se faire en harmonie avec la carte des productions agricoles. De ce qui précède l'on peut déduire qu'en principe, partout où l'on peut disposer d'eau en permanence, c'est-à-dire dans les zones guinéenne et forestière, la pisciculture est la bienvenue. Elle s'exclue des zones soudanaise et sahélienne où la pêche est le plus souvent active et où le régime des eaux, non permanent, ne permet pas d'envisager la construction aisée d'étangs, sauf dans un cas sur lequel nous reviendrons tout à l'heure.

\section{La Pisciculture.}

Il n'y a pas qu'une pisciculture dans l'Ouest africain, il y en a beaucoup. Avant toute action destinée à introduire la pisciculture, il convient de poser préalablement deux questions :

Avec quoi faire du poisson?

Quel poisson et comment?

Autrement dit, il faut confronter écologie et économie d'une part et technique d'autre part. De la technique nous dirons peu de chose, les publications qui en traitent sont nombreuses.

On peut avancer qu'actuellement la manière de construire des étangs rustiques avec barrages en terre compactée ne pose plus de problème particulier et qu'elle peut être adaptée à tous les besoins, ceux des collectivités comme ceux des familles. Par contre, la technique de la production du poisson est encore susceptible d'amélioration.

En raison de la rapidité de la reproduction de l'espèce, la pisciculture du Tilapia présente l'inconvénient d'une trop grande facilité apparente, laquelle semble autoriser beaucoup de fantaisies de la part du pisciculteur. Or, la plupart de ces fantaisies ont pour conséquence des rendements déplorables. Cependant, l'on sait maintenant, par une méthode simple, produire des Tilapia de taille marchande dans un délai relativement bref avec trois espèces éprouvées : Nilotica et Macrochir d'une part, Zilli d'autre part. La vulgarisation de cette technique est affaire de temps et de patience.

La chair du Tilapia de 80 à 100 grammes est très estimable pour les palais africains et européens. Ce fait est d'ailleurs connu depuis fort longtemps, peut-être même depuis l'antiquité, pour le Tilapia du Nil. Aussi, pourvu que l'étang produise une quantité raisonnable de poisson de la taille ci-dessus indiquée, le consommateur est satisfait si le poisson 
lui est présenté au sortir de l'eau. A cette condition, le Tilapia est préféré au poisson de mer conservé dans la glace, même si son prix est supérieur.

La pisciculture de l'Heterotis niloticus débute, mais elle donne de sérieux espoirs car l'Heterotis semble se reproduire bien plus tard que le Tilapia avec une croissance beaucoup plus rapide : Un Tilapia nilotica de 6 mois pesant 100 grammes est considéré comme un beau poisson, l'Heterotis de 6 mois atteint facilement 600 grammes et sa chair, quoique moins fine que celle du Tilapia nilotica est appréciable.

D'autres espèces seraient évidemment très dignes d'intérêt, en particulier les Clarias et le Parophiocephalus obscurus, ce dernier surtout, en raison de l'excellence de sa chair. Les uns comme les autres se reproduisent en étang, malheureusement leurs possibilités de respiration aérienne leur permettent de s'échapper des étangs et les rendent difficile à contrôler. Ils ne peuvent être considérés dans l'état actuel de nos connaissances, que comme espèces d'appoint à élever en même temps que le Tilapia ou l'Heterotis.

La question de la place de la pisciculture dans l'écologie et l'économie locales mérite d'être considérée avec quelque attention car, dans toute la nouveauté de la pisciculture du Tilapia, on a introduit celle-ci dans beaucoup d'endroits comme un miracle passe-partout, ce qui n'a pas été sans produire quelques mécomptes.

Comme il a été dit plus haut, dans la zone où l'on fait de la pisciculture, les eaux sont en général peu productives et ne servent que de " support " au poisson. Il faut sans doute de l'eau pour faire du poisson, mais il faut aussi autre chose.

La technique des engrais et amendements des étangs tropicaux est encore fort imparfaite; la liste des engrais naturels utilisables actuellement dans les étangs est brève et les engrais ou amendements artificiels sont en général trop chers pour une production qui est peu commercialisée. Plus intéressante est la possibilité de reproduire les meilleures conditions de productivité des eaux naturelles, c'est-à-dire de pratiquer l'assec et même l'assec cultivé si possible. Le riz est tout indiqué pour cette pratique et le maïs peut également utilisé, à condition de bien drainer les fonds.

En matière de nourriture du poisson, les possibilités sont, par contre, considérables. Sous le climat guinéen forestier, la production de matière verte, feuilles de tout genre, est très importante, d'où l'idée de la transformer en poisson. Une seule espèce s'est jusqu'à présent révélée apte à cet emploi : le Tilapia zilli. Encore ne consomme-t-il pas toutes les feuilles; il faut donc les choisir. Comme, d'autre part, le coefficient de transformation des feuilles en chair de poisson n'est pas très élevé, il faut en distribuer suffisamment. Ceci constitue, pour le pisciculteur, une lourde charge, laquelle n'est bien supportée que si l'étang est très 
facilement accessible et à proximité d'une " source " abondante de feuilles comestibles (colocase, patate, manioc le plus souvent).

Les Tilapia nilotica et macrochir ne peuvent être nourris qu'à partir de certains déchets de l'alimentation ou des industries alimentaires. Comme le coefficient de transformation de telles matières en poisson est beaucoup plus élevé que celui des feuilles, les quantités de nourriture à distribuer sont moindres. D'autre part, ces deux Tilapia microphages à l'état naturel, utilisent dans doute mieux le plancton que le Tilapia zilli. Cette faculté leur permet d'utiliser à la fois la nourriture par absorption directe et par l'intermédiaire du plancton.

Les sous-produits de l'industrie alimentaire africaine sont nombreux et variés : drèches de bières, son de céréales, graines de coton, épluchures de tubercules, tourteaux d'oléagineux, etc... L'éventail du choix est donc assez large, à condition naturellement que l'étang soit suffisamment proche de l'endroit d'où viennent les déchets.

L'Heterotis niloticus est microphage, mais aussi granivore. Les conditions optima de son élevage peuvent être liées principalement à la possibilité de distribution de graines de coton ou de brisures d'arachides, mais il semble qu'il puisse s'accomoder des produits utilisés pour nourrir les Tilapia macrochir et nilotica.

Ainsi donc, la pisciculture peut être pratiquée dans des conditions assez variables. C'est précisément la raison pour laquelle, avant de lancer une pisciculture, il importe de définir strictement les ressources en nourriture dont on peut disposer, ce qui détermine le choix de l'espèce à élever. Autrement dit, la pisciculture n'existe pas en soi : elle n'est qu'un maillon de la chaîne alimentaire locale. Quelquefois même, la pisciculture peut s'insérer dans le cycle d'assolement des cultures, lorsque l'on pratique l'assec cultivé en riz ou en maïs. Cette pratique est à la fois bénéfique pour le poisson et pour la culture. Elle permettrait peutètre de fixer certaines cultures itinérantes dévastatrices de galeries forestières en zone soudano-guinéenne, au grand bénéfice de la conservation des sols.

Dans le cadre de la conservation des sols, et cette fois ci, même en dehors de la zone classique de pisciculture, dans les zones soudanaises et soudano-sahélienne, les services forestiers et agricoles de certains pays ont entrepris la création de barrages de retenue d'eau sur la nappe phréatique ou sur des thalwegs asséchés en saison sèche. Outre l'intérêt de créer des points d'eau empoissonnables et des abreuvoirs, l'eau de certains de ces barrages peut-être utilisée pour des cultures irriguées et peut-être même pour une pisciculture de cycle court de saison sèche. On peut ainsi récupérer à la fois pour la culture et la pisciculture des terres complètement inutilisées.

De tous ces aspects annexes de la pisciculture, l'on peut conclure que le développement de celle-ci est lié à un plan d'aménagement rationnel des Territoires. Or, de tels plans n'existent en général qu'à l'état embryonnaire dans l'Ouest africain où, comme nous l'avons dit plus haut, toute la politique agricole est centrée sur le rapport immédiat 
en argent. Politique à courte vue, s'il en fut jamais et qu'il serait souhaitable de voir modifier au plus vite au moyen d'une étroite coordination des actions agricoles (sensu stricto), forestière, piscicole et zootechnique.

\section{La Pêche.}

A côté de l'immense action à entreprendre sous la rubrique "Pisciculture " quelque peu élargie, l'action sur la pêche apparaît plus restreinte. Il s'agit ici plutôt de récolter que de produire, ce qui est d'autant plus simple que les populations de pêcheurs ont, en général, des techniques de pêche assez élaborées qu'il suffit de prendre pour base de départ.

D'une manière générale, pour caractériser les améliorations possibles, on peut avancer :

- Que les pêcheurs sont en règle générale très sous-équipés en engins de pêche de bonne qualité; utilisé.

- Que le produit de la pêche est dans une très large mesure mal

De la première proposition, jointe à l'insuffisante concentration des populations de pêcheurs, il semble que l'on pourrait déduire que les plans d'eau africains sont sous-exploités. Ceci est probablement vrai dans la majorité des cas. Toutefois, des enquêtes statistiques devraient pouvoir le confirmer.

Il n'y a donc pas, semble-t-il, grand risque à équiper les pêcheurs pour augmenter leur pourcentage de travail utile. Pour ce faire, il n'est, en général, pas besoin de système compliqué de prêts : il suffit, après étude et démonstration préalables, de mettre les moyens nouveaux à leur disposition pour amorcer le circuit commercial des moyens de production, car la profession de pêcheur est le plus souvent lucrative. Mais, encore maintenant, dans de nombreuses régions, les populations de pêcheurs sont complètement en dehors des circuits économiques connus officiellement.

Les lieux de production du poisson d'eau douce ou saumâtre sont le plus souvent éloignés des lieux de consommation. Des procédés adéquat de conservation du produit sont donc nécessaires. Ceux-ci sont limités au séchage et au fumage, le caractère artisanal de l'industrie de la pêche et sa dispersion excluant la conservation par la glace au moins pour le moment.

Même pratiqués dans les conditions optima, séchage comme fumage supposent déjà une notable déperdition de matière consommable. Les techniques employées par les producteurs et par les commerçants aggravent ces inconvénients par l'imperfection des méthodes. Des améliorations très simples portant sur la conduite du séchage et du fumage, l'emballage et surtout la désinsectisation peuvent être appliquées avec grand profit. La principale difficulté consiste à trouver et à mettre en place un personnel convenable pour assurer la vulgarisation de ces améliorations. 
De même que pour la pisciculture, l'amélioration de l'industrie de la pêche ne se conçoit qu'en liaison étroite avec l'ensemble de l'économie régionale. La pêche donne une production infiniment plus importante que l'on ne se l'imaginait couramment mais les circuits commerciaux du poisson sont encore souvent bien difficiles à débrouiller. Aussi les répercussions des améliorations apportées à cette production risquent, au début, de n'être qu'imparfaitement appréciées.

Vus sous l'aspect décrit ci-dessus, il apparaît que les travaux sur la pêche proprement dite, ne donnent pas lieu à de grosses dépenses de vulgarisation. Les dépenses à consacrer aux études et enquêtes économiques semblent devoir être plus élevées. Mais, dans l'action piscicole, les dépenses importantes apparaissent dans l'aspect " Pisciculture ». Là, en effet, il s'agit non seulement de poisson, mais d'aménagement ou de récupération des terres pour utiliser au mieux les possibilités élevées de l'eau et du sol intertropical combinées.

La terre africaine est actuellement peu chargée en hommes, mais il faut prévoir que la poussée démographique mondiale peut changer dans un avenir assez proche cette situation et se préparer à y faire face. Une tâche de cette ampleur mérite l'attention, non seulement des pays africains eux-mêmes, mais de tous les pays qui ont pris intérêt à l'Afrique de l'Ouest.

Note : Depuis que cet article a été écrit (1958), la pisciculture de l'Heterotis niloticus, répondant aux espoirs que l'on avait fondés sur ce poisson, s'est développée dans certains pays de l'Ouest africain. Les pisciculteurs africains ont apprécié particulièrement le fait que l'Heterotis pouvait atteindre un poids élevé dans un temps relativement court (on a pu, dans certains cas, obtenir en moins d'un an des poissons avoisinant 3 kilogrammes à partir d'alevins de 100 grammes). Ceci permet d'obtenir sinon des productions globales plus importantes, du moins des valeurs à l'unité de produit beaucoup élevées qu'avec l'élevage du Tilapia.

D'autre part la pisciculture du Tilapia, elle aussi, a progressé et l'on sait maintenant éliminer, soit par la culture monosexe, soit par l'utilisation de Prédateur, l'excès de production d'alevins qui était le principal inconvénient de cette pisciculture.

(Septembre 1960). 